\title{
GCU
}

Glasgow Caledonian

University

University for the Common Good

\section{Effectiveness of social network interventions to support cardiac rehabilitation and secondary prevention in the management of people with heart disease}

Purcell, Carrie; Smillie, Susie; Hilton Boon, Michele; Simpson, Sharon; Taylor, Rod

Published in:

Cochrane Database of Systematic Reviews

DOI:

10.1002/14651858.cd013820

Publication date:

2021

Document Version

Publisher's PDF, also known as Version of record

Link to publication in ResearchOnline

Citation for published version (Harvard):

Purcell, C, Smillie, S, Hilton Boon, M, Simpson, S \& Taylor, R 2021, 'Effectiveness of social network interventions to support cardiac rehabilitation and secondary prevention in the management of people with heart disease', Cochrane Database of Systematic Reviews, vol. 2021, CD013820.

https://doi.org/10.1002/14651858.cd013820

\section{General rights}

Copyright and moral rights for the publications made accessible in the public portal are retained by the authors and/or other copyright owners and it is a condition of accessing publications that users recognise and abide by the legal requirements associated with these rights.

Take down policy

If you believe that this document breaches copyright please view our takedown policy at https://edshare.gcu.ac.uk/id/eprint/5179 for details

of how to contact us. 
Cochrane Database of Systematic Reviews

\section{Effectiveness of social network interventions to support cardiac rehabilitation and secondary prevention in the management of people with heart disease (Protocol)}

Purcell C, Smillie S, Hilton Boon M, Simpson SA, Taylor RS

Purcell C, Smillie S, Hilton Boon M, Simpson SA, Taylor RS.

Effectiveness of social network interventions to support cardiac rehabilitation and secondary prevention in the management of people with heart disease (Protocol).

Cochrane Database of Systematic Reviews 2020, Issue 12. Art. No.: CD013820.

DOI: $10.1002 / 14651858 . C D 013820$.

www.cochranelibrary.com

Effectiveness of social network interventions to support cardiac rehabilitation and secondary prevention in the management of people with heart disease (Protocol) 
TABLE OF CONTENTS

HEADER 1

ABSTRACT

BACKGROUND

OBJECTIVES

METHODS

ACKNOWLEDGEMENTS

REFERENCES

APPENDICES

HISTORY

CONTRIBUTIONS OF AUTHORS

DECLARATIONS OF INTEREST

SOURCES OF SUPPORT 
[Intervention Protocol]

\section{Effectiveness of social network interventions to support cardiac rehabilitation and secondary prevention in the management of people with heart disease}

Carrie Purcell ${ }^{1}$, Susie Smillie ${ }^{1}$, Michele Hilton Boon ${ }^{1}$, Sharon A Simpson ${ }^{1}$, Rod S Taylor ${ }^{2}$

$1 \mathrm{MRC} / \mathrm{CSO}$ Social and Public Health Sciences Unit, University of Glasgow, Glasgow, UK. 2MRC/CSO Social and Public Health Sciences Unit \& Robertson Centre for Biostatistics, Institute of Health and Well Being, University of Glasgow, Glasgow, UK

Contact address: Carrie Purcell, carrie.purcell@glasgow.ac.uk.

Editorial group: Cochrane Heart Group.

Publication status and date: New, published in Issue 12, 2020.

Citation: Purcell C, Smillie S, Hilton Boon M, Simpson SA, Taylor RS. Effectiveness of social network interventions to support cardiac rehabilitation and secondary prevention in the management of people with heart disease (Protocol). Cochrane Database of Systematic Reviews 2020, Issue 12. Art. No.: CD013820. DOI: 10.1002/14651858.CD013820.

Copyright @ 2020 The Cochrane Collaboration. Published by John Wiley \& Sons, Ltd.

\section{A B S T R A C T}

\section{Objectives}

This is a protocol for a Cochrane Review (intervention). The objectives are as follows:

To assess the effectiveness of social network and social support interventions to support cardiac rehabilitation and secondary prevention in the management of people with heart disease.

As a secondary output of this review, and to assist in conceptualising future research focused on social network and social support interventions, we aim to develop a logic model theorising the relationship between social networks or social support and heart disease outcomes. We will draw on existing models of social support for health (e.g. Berkman 2000), as well as established approaches to theorising and implementing behaviour change (e.g. Michie 2011). 


\section{B A C K G R O U N D}

\section{Description of the condition}

'Heart disease' encompasses a range of disorders affecting the heart, including: diseases of the heart blood vessels (coronary heart disease (CHD)); heart rhythm problems (arrhythmias, such as atrial fibrillation); heart infections; and congenital heart defects. CHD is the most common type of heart disease; its common symptoms are chest pain (angina) and heart attack (myocardial infarction). Coronary heart disease may necessitate a revascularisation procedure, and can lead to chronic heart failure. Globally, cardiovascular diseases (CVD; heart and circulatory diseases combined) contribute to $31 \%$ of all deaths, more than any other cause (WHO 2017). Of the estimated 17.7 million deaths from CVD in 2015, the most common cause (7.4 million) was CHD. Of 17 million premature deaths from non-communicable diseases in the same year, $37 \%$ were caused by CVD (WHO 2017).

Cardiac rehabilitation (CR) programmes aim to go beyond secondary prevention and address physical, mental, and social factors, to support individuals to continue or resume their dayto-day life following a cardiac event, diagnosis, or procedure (BACPR 2017). CR programmes are widely offered, in line with guidance in the UK and globally, to people with a number of presentations of heart disease, and include various psychosocial, educational, health behaviour change, and risk management components (Dalal 2015 ). In this review, we will focus on heart disease indications for which people in the United Kingdom are typically referred for cardiac rehabilitation, namely: CHD (including myocardial infarction, post-revascularisation, and stable angina); chronic heart failure; atrial fibrillation; and following valve replacement or repair procedures (BACPR 2017).

\section{Description of the intervention}

The impact on health behaviours of social networks, and the social support communicated through them, has generated a vast body of literature, grounded in sociological, anthropological, and psychological theory (Berkman 2000). Interest has particularly increased in recent times in relation to how the influence of social networks might be harnessed in health interventions. Evidence suggests that social network or social support interventions are associated with positive outcomes in some health behaviours (Hunter 2019). Social support or social network interventions may have potential in rehabilitation or secondary prevention programmes for CVD, since low levels of social support are specifically associated with increased risk of CHD events (Lett 2005), and social isolation and weak social networks have been linked to poor outcomes for individuals with heart disease (Heidari Gorji 2019). Contemporary technologies facilitate remote provision or engendering of social support, for example the use of social media, smart device applications (apps), video conferencingbased interventions, potentially broadening the scope of social network interventions for health. As such, social network and social support interventions may play an important role as an adjunct to current evidence-based secondary prevention and rehabilitation measures, in improving outcomes for people with CVD (Piepoli 2016).

The scope of this review includes interventions specifically identified as 'social network' or 'social support' interventions. Diversity in how these terms are understood and used means conceptual clarity is essential. In this review, and following Berkman 2000, we will use 'social network' to mean 'the web of social relationships that surrounds an individual and the characteristics of those ties' (e.g. family, friendship, or other social groups); and 'social support' to mean one of the 'mediating pathways by which networks might influence health', using emotional, instrumental, appraisal, or informational means (e.g. via peer-to-peer support, app-based support, dedicated online groups). We will use 'social network' or 'support intervention' to indicate interventions that explicitly aim to mobilise relationships with partners, caregivers, friends, family members, or peer supporters (e.g. others with the same condition), to improve health.

The review will include interventions that capitalise on social networks or social support, which may be implemented individually or in groups, and in a range of settings including home, primary or secondary care, or remotely (by phone or online). The review will include interventions which: aim to reduce social isolation or improve connectedness, or both; use apps or social media technologies to connect people with heart disease; or use a formal component of Social Network Analysis (SNA); or a combination. We do not intend to include studies where SNA has been used in study methodology, but where the intervention itself does not involve elements of social support, or where social media platforms are used without an intended element of social support.

\section{How the intervention might work}

Social networks and social support may operate at a number of levels to promote healthy behaviours, through mechanisms including: reinforcement, encouragement, motivation, feedback, empathy, role modelling, increased self-efficacy, instrumental support (i.e. practical help), emotional support, appraisal (e.g. affirmation), peer pressure for healthy behaviours, or access to health information (Simpson 2015). Social support can be provided by family, friends, peers or wider social networks, and members of specific groups with a common behavioural goal (e.g. weight loss groups). Social support is identified as a key component in both initiating and sustaining behaviour change (Hunter 2019; Simpson 2011; Simpson 2020). Nevertheless, how social networks or social support might improve outcomes for people with heart disease is largely under-theorised.

The application of behaviour change theory in a different health context (weight loss) suggests a social network or social support intervention can facilitate goal setting, action planning, problem solving, support ongoing health goals, encourage self-monitoring, and promote autonomy (Simpson 2020). They may offer an opportunity to enhance inclusion and accessibility for populations who are less likely to engage in cardiac rehabilitation (CR), such as women and people from black, Asian, and minority ethic (BAME) groups (Dalal 2015 ). Contextual factors, which might impact the feasibility and effectiveness of social network or social support interventions might include: the availability of friends, family, or peers to offer support; characteristics of the person offering support; characteristics of the social network or relationship; degree of integration within a social network; access to resources (e.g. time to exercise, healthy food, etc.); socioeconomic factors; and everyday barriers to, and facilitators of participation (Berkman 2000; Simpson 2011).

Effectiveness of social network interventions to support cardiac rehabilitation and secondary prevention in the management of people 


\section{Why it is important to do this review}

Heart disease is a global public health concern associated with significant health, social, and economic burden in developed and developing countries alike. Social isolation and low social support have been shown to be common in people with cardiac disorders, and associated with poor outcomes, including increased risk of hospital readmission (Heidari Gorji 2019; Lett 2005; Mookadam 2004). More broadly, there is growing interest in understanding the impact of social network and social support-based interventions on health-related behaviour (Berkman 2000). Interventions with social support components are not well understood, and there is no current consensus on how they might be most effectively provided.

The addition of social network or social support interventions may have a role in enhancing the effectiveness of secondary prevention and $C R$. In the UK and globally, CR is widely offered for the conditions noted above, and includes various psychosocial, educational, health behaviour change, and risk management components (Dalal 2015 ). However, uptake is suboptimal and inequitable - due to a combination of factors including accessibility, dislike of centre-based classes, an absence of both uniform referral processes and physician familiarity, and availability - with disproportionately lower referral and uptake in women, and people in lower socioeconomic, or BAME groups (Dalal 2015 ). In some contexts, up-front costs to users of $C R$ and inconsistent reimbursement also act as barriers (Babu 2016). Lower cost, non-centre-based CR can improve uptake (Dalal 2019). However, depending on the mode of delivery, it may risk limiting face-to-face interaction with others with similar conditions. Including social network or social support components to $\mathrm{CR}$ programmes may address this limitation. Guidelines on CR specifically highlight the importance of improving outcomes by using existing social support, and encouraging new social connections where available and appropriate (BACPR 2017; SIGN 2017). They also highlight the potential to capitalise on new technologies (Piepoli 2016). As a result of the COVID-19 pandemic, the need for secondary prevention and effective models of CR, based outside traditional clinical settings - including in the home and using remote delivery - has become acute, as has the need to minimise social isolation. The current context highlights challenges, opportunities, and the demand for innovative approaches, including social network and social support interventions (Dalal 2020; de Sire 2020).

By conducting this review, we aim to contribute to a body of knowledge on the potential benefits of interventions that use or facilitate social networks or social support for people with heart disease, and to inform future research and intervention development.

\section{OB JECTIVES}

To assess the effectiveness of social network and social support interventions to support cardiac rehabilitation and secondary prevention in the management of people with heart disease.

As a secondary output of this review, and to assist in conceptualising future research focused on social network and social support interventions, we aim to develop a logic model theorising the relationship between social networks or social support and heart disease outcomes. We will draw on existing models of social support for health (e.g. Berkman 2000), as well as established approaches to theorising and implementing behaviour change (e.g. Michie 2011).

\section{METHODS}

\section{Criteria for considering studies for this review}

\section{Types of studies}

We will include randomised controlled trials (with individual participant allocation, cluster-allocation, or cross-over design) of a social network or social support intervention for people with heart disease. We will include trials regardless of their duration of followup, and we will include studies reported as full text, those published as abstract only, and unpublished data.

\section{Types of participants}

We will include all individuals, aged $\geq 18$ years with physiciandiagnosed: chronic heart failure (hereafter 'heart failure'), coronary heart disease (myocardial infarction (MI), post-revascularisation, or stable angina), atrial fibrillation, or post-valve replacement or repair procedures.

If a trial contains a mixed population (i.e. individuals with diagnoses other than heart disease) we will (1) assess whether subgroup results for heart disease are reported. If not, we will contact the trial authors. If results are not available, we will (2) include all the trial data as long as people with heart disease make up 50\% or more of the total trial population. We will carry out a sensitivity analysis to assess the impact of including studies with mixed populations, if we are unable to get the subgroup data from trialists.

We will exclude trials if they do not focus (at least $50 \%$ of trial population) on participants with heart failure, coronary heart disease (MI, post-revascularisation, or stable angina), atrial fibrillation, or post-valve replacement or repair procedures.

\section{Types of interventions}

We will include all interventions that explicitly include a component of social network or social support, i.e. interventions that involve partners, caregivers, friends, family members, peer or lay supporters (e.g. others with the same condition). Interventions may be implemented individually or in groups, including those using applications (apps) or other social media technologies to connect people with heart disease. We will not include interventions where the only form of social support is interaction with a healthcare or social work professional (even where framed explicitly as 'social support'). We will consider all social network or social support interventions, delivered either alone or as part of a wider cardiac rehabilitation (CR) programme (e.g. with exercise or educational components), i.e. trial comparison of CR plus social network or social support intervention versus $\mathrm{CR}$ alone. The intervention may be based in any setting, including the participant's home, the community, or in primary or secondary care, providing the above criteria are met. Interventions may be supervised or unsupervised, and single or multi-component.

The review will characterise the nature of the intervention (e.g. duration, intensity, mode of delivery) for each included study. If there are sufficient studies, we will explore the impact of these intervention variations on participant outcomes, using approaches that include meta-regression. 
Both the intervention and control group participants will receive usual medical care as reported by the study. Usual care typically comprises secondary prevention measures, including regular check-ups, drug treatment (e.g. antihypertensive or lipid-lowering drugs), and advice for a healthy and active lifestyle (e.g. diet, smoking, and physical activity). Some people may also receive a programme of CR. This review will include studies that explicitly assesses the impact of the addition of a social network or social support intervention to a usual care comparator that could include secondary prevention alone, or secondary prevention and CR.

We recognise that trial participants may be receiving cointerventions (e.g. antihypertensive, lipid-lowering therapy), and we anticipate that the receipt of such co-interventions will be balanced across intervention and control arms.

\section{Types of outcome measures}

Reporting one or more of the outcomes listed here in the trial is not an inclusion criterion for the review. When a published report does not appear to report one of these outcomes, we will access the trial protocol and contact the trial authors to ascertain whether the outcomes were measured but not reported. We will include relevant trials that measured these outcomes but did not report the data at all, or not in a usable format, as part of the narrative in the review.

We will extract primary and secondary outcomes at all reported follow-up points, categorised as short-term (12 months or less) or long-term (more than 12 months). As long-term follow-up (more than 12 months) is our period of most interest - due to its usefulness in informing policy decisions - we will seek to include the longest follow-up period in the 'Summary of findings' table, depending on the duration of follow-up in the included trials.

\section{Primary outcomes}

1. All-cause mortality

2. Cardiovascular-related mortality

3. All-cause hospital admission (number of events)

4. All-cause hospital admission (number of participants with at least one event)

5. Cardiovascular-related hospital admission (number of events)

6. Cardiovascular-related hospital admission (number of participants with at least one event)

7. Health-related quality of life (HRQoL) assessed by validated generic (e.g. Short-Form 36 (SF-36)) or disease specific (e.g. HeartQoL) measures

\section{Secondary outcomes}

1. Psychological well-being (validated measures of depression and anxiety e.g. HADS)

2. Heart disease risk factors:
a. smoking,
b. blood pressure (systolic),
c. blood pressure (diastolic),
d. LDL,
e. HDL, and
f. total cholesterol

3. Myocardial infarction (number of events)
4. Myocardial infarction (number of participants with at least one event)

5. Revascularisation (number of events)

6. Revascularisation (number of participants with at least one event)

7. Physical activity behaviour (validated self-report measures of physical activity e.g. IPAQ-SF, GPAQ, and EHIS-PAQ, or objective measures like accelerometry)

8. Return to work or full-time education

9. Social isolation and connectedness using validated outcomes (e.g. Multidimensional Scale of Perceived Social Support (MSPSS), Personal Social Capital Scale (PSCS))

10.Participant satisfaction (validated measures, e.g. Patient Satisfaction Questionnaire)

11.Adverse events: we will include any reports of adverse events related to the intervention, by number of events of each type. We define adverse events as any untoward occurrence related to the intervention (e.g. psychological distress associated with use of the intervention). We will classify adverse events as 'serious' (any occurrence that can result in life-threatening situations, disability, or death, or requires hospitalisation of any duration or 'non-serious' (European Commission 2011).

We will not include cost as a specific outcome. However, should such data be available, we will comment on them in a narrative format.

\section{Search methods for identification of studies}

\section{Electronic searches}

We will identify trials through systematic searches of the following bibliographic databases:

- Cochrane Central Register of Controlled Trials (CENTRAL) in the Cochrane Library;

- MEDLINE Ovid (1946 onwards);

- Embase Ovid (1980 onwards);

- Web of Science Core Collection.

We will adapt the preliminary search strategy for MEDLINE Ovid to use in the other databases (Appendix 1). We will apply the Cochrane sensitivity and precision-maximising RCT filter to MEDLINE Ovid, and adaptations of it to the other databases, except CENTRAL (Lefebvre 2019).

We will conduct a search of the US National Institutes of Health Ongoing Trials Register ClinicalTrials.gov (www.ClinicalTrials.gov), and the World Health Organization International Clinical Trials Registry Platform (apps.who.int/trialsearch/) for ongoing or unpublished trials.

We will search all databases from their inception to the present, and impose no restriction on language of publication or publication status. We will not perform a separate search for adverse effects of social network or social support interventions for people with heart disease.

\section{Searching other resources}

We will check reference lists of all included studies and any relevant systematic reviews identified, for additional references to trials. We will also examine any relevant retraction statements and errata for

Effectiveness of social network interventions to support cardiac rehabilitation and secondary prevention in the management of people 
included studies. We will contact the main authors of studies and experts in the field to ask for any missed, unreported, or ongoing trials.

\section{Data collection and analysis}

\section{Selection of studies}

Two review authors (CP and SS) will independently screen the titles and abstracts of all records we identify as a result of the search, and code them as 'retrieve' (eligible, or potentially eligible, or unclear), or 'do not retrieve'. If there are any disagreements, they will ask a third review author to arbitrate (RST or SAS). We will retrieve the full-text study reports and publication, and two review authors (CP and SS) will independently screen the full-text report, identify studies for inclusion, and identify and record reasons for excluding the ineligible studies. We will resolve any disagreement through discussion, or if required, we will consult a third person (RST or SAS). We will identify and exclude duplicates, and collate multiple reports of the same study, so that each study rather than each report is the unit of interest in the review. We will record the selection process in sufficient detail to complete a PRISMA flow diagram and 'Characteristics of excluded studies' table (Liberati 2009).

\section{Data extraction and management}

We will use a data collection form for study characteristics and outcome data, which has been piloted on at least one study in the review. One review author (CP and SS) will extract the following study characteristics from included studies.

1. Methods: study design, total duration of study, number of study centres and location, study setting, and date of study

2. Participants: $\mathrm{N}$ randomised, $\mathrm{N}$ lost to follow-up or withdrawn, $\mathrm{N}$ analysed, mean age, age range, gender, heart disease diagnosis, inclusion criteria, and exclusion criteria

3. Interventions: intervention, comparison, and co-interventions. The intervention description will include coding of the nature of intervention delivery categorised, for example by: behaviour change techniques used; dose; type of support provided who provides support (e.g. partners, family, peer supporters); whether face-to-face or remote, delivered one-to-one or in a group, supervised or unsupervised, etc.

4. Outcomes: primary and secondary outcomes specified and collected, and time points reported

5. Notes: funding for trial, and notable conflicts of interest of trial authors

Two review authors (CP and SS) will independently extract outcome data from included studies. We will resolve disagreements by consensus, or by involving a third person (SAS or RST). One review author (CP) will transfer data into RevManWeb (Review Manager 2020). We will double-check that data are entered correctly by comparing the data presented in the systematic review with the data extraction form. A second review author (SS) will spot-check study characteristics for accuracy against the trial report.

\section{Assessment of risk of bias in included studies}

Two review authors (MHB and either SS or $\mathrm{CP}$ ) will independently assess risk of bias for each study using the second version of the Cochrane 'Risk of bias' tool (RoB 2), outlined in the Cochrane Handbook for Systematic Reviews of Interventions (Higgins 2019c).
We will resolve any disagreements by discussion, or by involving another review author (RST or SAS). The effect of interest we will assess is the effect of assignment to the intervention. The outcomes we will assess are those included in the 'Summary of findings' table. We will use the 'RoB 2' tool to manage the assessment of bias. We will assess the risk of bias of specific results of a trial according to the following domains:

1. bias arising from the randomisation process;

2. bias due to deviations from intended interventions;

3. bias due to missing outcome data;

4. bias in measurement of the outcome; and

5. bias in selection of the reported result.

For cluster-RCTs we will add an additional domain, $1 \mathrm{~b}$, 'Bias arising from the timing of identification and recruitment of participants', and use the signalling questions from the archived version of the 'ROB 2' tool. For cross-over RCTs, we will use the signalling questions specific to cross-over trials from the archived version of the 'ROB 2' tool for domain 2, and for signalling question 3.2 of domain 3.

We will assess risk of bias in each domain. An algorithm (decision tree) using a series of signalling questions and answers (yes, probably yes, no information, probably no, no) will determine risk of bias (low risk, some concerns, and high risk).

Our analysis of bias due to deviations from intended interventions, will assess the effect of assignment to the intervention at baseline, sometimes known as the 'intention-to-treat effect'.

We will classify each potential source of bias (as low, high, or some concerns), and provide a quotation from the study report, and a justification for our judgement, recording these details in the Excel 'ROB 2' tool. We will summarise our 'Risk of bias' judgements across different studies, for each of the domains listed. Where information on risk of bias relates to unpublished data or correspondence with a trial author, we will note this in the Excel 'ROB 2' tool.

When analysing treatment effects, we will consider the risk of bias for the studies that contribute to that outcome. In the Results section of the review, we will present visual summaries of the 'ROB 2 ' judgments for each outcome and study, and narrative summaries of the 'Risk of bias' judgments for each domain within each outcome. We will provide full details of the consensus decisions on risk of bias for each signalling question, study, and outcome, together with supporting quotations, in an online repository. We will determine the overall risk of bias for each study, according to the criteria for reaching overall 'Risk of bias' judgments, set out in Table 8.2.b in the Handbook (Higgins 2019c).

\section{Measures of treatment effect}

We will analyse dichotomous data as risk ratios (RR) with 95\% confidence intervals $(\mathrm{Cl})$, and continuous data as mean difference (MD) with 95\% confidence intervals. For any outcomes that are measured by studies in a variety of ways, we will either analyse these outcomes separately, or we will use the standardised mean difference (SMD) with 95\% confidence intervals as a summary statistic. We will interpret the SMD using the two approaches recommended in the Handbook. First, for all mean pooled SMD, we will apply the rule of thumb, based on Cohen's effect sizes, as advised in the Handbook: i.e. 0.2 represents a small effect, 0.5 a

Effectiveness of social network interventions to support cardiac rehabilitation and secondary prevention in the management of people 
moderate effect, and 0.8 a large effect. In addition, we will convert the SMD to the original scale units, by multiplying the pooled mean SMD by a standard deviation (SD) from a particular trial. We will enter data presented as a scale with a consistent direction of effect. We will describe skewed data, reported as medians and interquartile ranges, in a narrative format.

\section{Unit of analysis issues}

In accordance with Section 16.4 of the Cochrane Handbook for Systematic Reviews of Interventions, we will aim to include data from both periods of any cross-over trials identified, assuming (i) there was a wash-out period considered long enough to reduce carry-over, (ii) no irreversible events, such as mortality occurred, and (iii) appropriate statistical approaches were used (Higgins 2019a).

We will use multiple time points from individual trials; to define completely separate pooled analyses of outcomes (e.g. HRQoL less than six months; HRQoL between six and 12 months, etc.). This approach will avoid the situation in which the same data appear more than once in the same analysis.

We will adjust cluster-RCT sample sizes or standard errors using the methods outlined in the Cochrane Handbook for Systematic Reviews of Interventions, using an estimate of the intra-cluster correlation co-efficient (ICC) derived from the trial, from a similar trial, or from a study of a similar population. If we use ICCs from other sources, we will report this result, and conduct sensitivity analyses to investigate the effect of variation in the ICC.

If a trial has more than two control or intervention arms (e.g. cardiac rehabilitation and social support (intervention arm) versus cardiac rehabilitation alone (control arm 1 ), versus no intervention (control arm 2)), we will include two interventions versus control comparisons, by dividing the number randomised to the intervention group for each comparison in half to obtain the denominator for data analysis; the mean and standard deviation for the intervention group remain unchanged for both comparisons. We will also acknowledge heterogeneity in the randomisation unit, and conduct a subgroup analysis to investigate the effects of the randomisation unit.

\section{Dealing with missing data}

We will contact investigators or study sponsors in order to verify key study characteristics and obtain missing numerical outcome data, where possible (e.g. when a study is identified as abstract only). Where possible, we will use RevManWeb calculator to calculate missing standard deviations, using other data from the trial, such as confidence intervals, based on methods outlined in the Handbook (Higgins 2019b).

\section{Assessment of heterogeneity}

We will inspect forest plots visually to consider the direction and magnitude of effects, and the degree of overlap between confidence intervals. We will use the $I^{2}$ statistic to measure heterogeneity among the trials in each analysis, but acknowledge that there is substantial uncertainty in the value of $I^{2}$ when there is only a small number of studies. We will also consider the $P$ value from the $\mathrm{Chi}^{2}$ test, i.e. the confidence interval for $\mathrm{I}^{2}$. If we identify considerable heterogeneity, i.e. $I^{2}$ values of $75 \%$ to $100 \%$, we will report it and explore possible causes by pre-specified subgroup analysis. We will also explore clinical heterogeneity of included studies qualitatively, by comparing the characteristics of included studies.

\section{Assessment of reporting biases}

If we are able to pool more than 10 trials, we will create and examine a funnel plot, and use the Egger test, to explore possible small study biases for the primary outcomes (Egger 1997).

\section{Data synthesis}

We will include all eligible studies in the primary analysis. We will undertake meta-analyses only where this is meaningful, i.e. if the interventions, participants, and outcomes are similar enough for pooling to make sense.

We will express dichotomous outcomes for each comparison as risk ratios (RR). We will express continuous data as mean difference (MD) with $95 \% \mathrm{Cl}$; or, when an outcome is measured and reported in more than one way (e.g. HRQoL), as standardised mean difference (SMD) with $95 \% \mathrm{Cl}$. We will enter data presented as a scale with a consistent direction of effect.

Given the likely clinical heterogeneity in trials we will include in this review, where it is judged appropriate to formally pool studies, we will initially use a random-effects meta-analysis model. For all meta-analyses, we will also use a fixed-effects model, because of the tendency of smaller trials (which are more susceptible to publication bias) to be over-weighted with a random-effects analysis; we will comment when there are differences between results from fixed-effect and random-effects models (Heran 2008a; Heran 2008b). We will complete data synthesis and analyses using RevMan Web (Review Manager 2020). We will conduct metaregression analysis using the 'metareg' command in Stata version 16.060 (Stata).

To refine the proposed theory of change, and to assess the plausibility of the theorised causal pathways, we will map the results of the data synthesis against our initial logic model.

\section{Subgroup analysis and investigation of heterogeneity}

We anticipate the size of effect for some outcomes will be related to the length of the follow-up. Therefore, we will present separate meta-analyses of each outcome according to length of follow-up: i.e. we will pool studies with short-term follow-up (12 months or less), and long-term follow-up (more than 12 months), based on the longest follow-up reported. We will also try to undertake univariate meta-regression, to explore heterogeneity and examine potential treatment effect modifiers. For the primary outcomes, we will aim to test whether a relationship exists between effect estimates and the following study characteristics:

1. Risk of bias i.e. high risk or high risk/some concerns versus low risk of bias

2. Type of intervention (e.g. social network or social support only versus multi-component intervention (categorical variable))

3. Duration of social network or social support intervention (continuous variable)

4. Type of population (e.g. acute event or procedure (post$\mathrm{MI}$, revascularisation, valve surgery) versus chronic condition (e.g. heart failure, atrial fibrillation, stable angina; (categorical variable))

Effectiveness of social network interventions to support cardiac rehabilitation and secondary prevention in the management of people 
5. Nature of intervention delivery (e.g. home versus centre-based supervised versus unsupervised, remote (phone or online) versus face-to-face; trained or qualified practitioner versus lay person delivery; one-to-one versus group; (categorical variable))

6. Study location (low- or middle-income countries (LMIC) or highincome countries (HIC), as per Organisation for Economic Cooperation and Development (OECD) classification; (categorical variable))

7. Mean age of participants (continuous variable)

8. Percentage of male and female participants (continuous variable)

Given the anticipated small ratio of trials to covariates, we will limit meta-regression to univariate analysis, and to situations in which 10 or more trials are available (Higgins 2019a). We anticipate a small number of included studies; therefore, we recognise that it is unlikely we will be able to conduct a meta-regression or a stratified meta-analysis. If reported by individual included studies, we will extract results of subgroup analyses, including participantlevel subgroup analyses; for example, if a trial reports whether there was a difference in the effectiveness of interventions between males and females. If applicable, we will use the formal test for subgroup differences in RevMan Web, and base our interpretation on this (Review Manager 2020).

\section{Sensitivity analysis}

If applicable, we will conduct sensitivity analyses to assess the impact of including studies with mixed populations (where subgroup data are not available from the trialists), and to investigate the effect of variation in the intra-cluster correlation coefficient (if ICCS from other sources are used).

\section{Summary of findings and assessment of the certainty of the evidence}

We will create a 'Summary of findings' table with our primary outcomes:

1. All-cause mortality

2. Cardiovascular-related mortality

3. All-cause hospital admission (number of events)

4. All-cause hospital admission (number of participants with at least one event)

5. Cardiovascular-related hospital admission (number of events)
6. Cardiovascular-related hospital admission (number of participants with at least one event)

7. Health-related quality of life (HRQoL) assessed by validated generic (e.g. Short-Form 36) or disease specific (e.g. HeartQoL) measures

\section{Adverse events}

We will use the five GRADE considerations (risk of bias, consistency of effect, imprecision, indirectness, and publication bias) to assess the certainty of a body of evidence as it relates to the studies that contribute data to the meta-analyses for the prespecified outcomes. We use the overall 'RoB 2' judgment for each outcome as part of the GRADE assessment. We will use methods and recommendations described in Chapter 14 of the Cochrane Handbook for Systematic Reviews of Interventions (Schünemann 2019), and use GRADEpro software (GRADEpro GDT). We will justify all decisions to downgrade the certainty of the evidence using footnotes and we will make comments to aid reader's understanding of the review where necessary.

Long-term follow-up (> 12 months) is our follow-up period of most interest, because it is useful to inform policy decisions. Therefore, we will include long-term follow-up (>12 months) for each of the specified outcomes in the 'Summary of findings' table.

Two review authors (CP and SS) will independently make judgements about the certainty of the evidence, with disagreements resolved by discussion, or by involving a third review author (RST or SAS). They will justify, and document their judgments, and incorporate them into the reporting of results for each outcome.

\section{ACKNOWLEDGEMENTS}

We gratefully acknowledge the Cochrane Heart Group for their support in the drafting of this protocol. In particular, we would like to thank Nicole Martin, Andrea Takeda, and Charlene Bridges, and the peer reviewers (Gordon McGregor, Gozde Ozakinci, Alexander M Clark, Brian SK Li) for their valuable feedback. We would also like to acknowledge the MRC/CSO Social and Public Health Sciences Unit, University of Glasgow, as the host institution for all the review authors with funding from the Medical Research Council (grant numbers MC_UU_12017/14 and MC_UU_12017/15) and Chief Scientist Office of the Scottish Government Health and Social Care Directorates (grant numbers SPHSU14 and SPHSU15). 


\section{RE F E R E N C E S}

\section{Additional references}

\section{Babu 2016}

Babu AS, Lopez-Jimenez F, Thomas RJ, Isaranuwatchai W, Herdy AH, Hoch JS, et al. Advocacy for outpatient cardiac rehabilitation globally. BMC Health Services Research 2016;16(471). [DOI: https://doi.org/10.1186/s12913-016-1658-1]

\section{BACPR 2017}

British Association for Cardiovascular Prevention and Rehabilitation. BACPR standards and core components for cardiovascular disease prevention and rehabilitation. www.bacpr.com/resources/ BACPR_Standards_and_Core_Components_2017.pdf (accessed prior to 16 October 2020).

\section{Berkman 2000}

Berkman LF, Glass T, Brissette I, Seeman TE. From social integration to health: Durkheim in the new millennium. Social Science \& Medicine 2000;51(6):843-57.

\section{Dalal 2015}

Dalal HM, Doherty P, Taylor RS. Cardiac rehabilitation. BMJ 2015;351:h5000.

\section{Dalal 2019}

Dalal HM, Taylor RS, Jolly K, Davis RC, Doherty P, Miles J, et al. The effects and costs of home-based rehabilitation for heart failure with reduced ejection fraction: the REACH$\mathrm{HF}$ multicentre randomized controlled trial. European Journal of Preventative Cardiology 2019;26(3):262-72. [DOI: $10.1177 / 2047487318806358]$

\section{Dalal 2020}

Dalal H, Taylor RS, Greaves C, Doherty PJ, McDonagh, ST, van Beurden SB, et al, REACH-HF Study Group. Correspondence to the EJPC in response to position paper by Ambrosetti $\mathrm{M}$ et al. 2020: cardiovascular rehabilitation and COVID-19: the need to maintain access to evidence-based services from the safety of home. European Journal of Preventive Cardiology 2020 April 28 [Epub ahead of print]. [DOI: 10.1177/2047487320923053]

\section{de Sire 2020}

De Sire A, Andrenelli E, Negrini F, Negrini S, Ceravolo MG. Systematic rapid living review on rehabilitation needs due to Covid-19: update to April 30th 2020. European Journal of Physical and Rehabilitation Medicine 2020 May 15 [Epub ahead of print]. [DOI: 10.23736/s1973-9087.20.06378-9]

\section{Egger 1997}

Egger M, Smith GD, Schneider M, Christoph M. Bias in metaanalysis detected by a simple, graphical test. BMJ 1997;315:629.

\section{European Commission 2011}

European Commission. Communication from the Commission - detailed guidance on the collection, verification and presentation of adverse event/reaction reports arising from clinical trials on medicinal products for human use ('CT-3'); 2011/C 172/01. Official Journal of the European
Union (eur-lex.europa.eu/LexUriServ/LexUriServ.do? uri=OJ:C:2011:172:0001:0013:EN:PDF).

\section{GRADEpro GDT [Computer program]}

McMaster University (developed by Evidence Prime) GRADEpro GDT. Version accessed prior to 5 November 2020. Hamilton (ON): McMaster University (developed by Evidence Prime). Available at gradepro.org.

\section{Heidari Gorji 2019}

Heidari Gorji MA, Fatahian A, Farsavian A. The impact of perceived and objective social isolation on hospital readmission in patients with heart failure: A systematic review and metaanalysis of observational studies. General Hospital Psychiatry 2019;60:27-36. [DOI: doi:10.1016/j.genhosppsych.2019.07.002]

\section{Heran 2008a}

Heran BS, Wong MM, Heran IK, Wright JM. Blood pressure lowering efficacy of angiotensin converting enzyme (ACE) inhibitors for primary hypertension. Cochrane Database of Systematic Reviews 2008, Issue 4. Art. No: CD003823. [DOI: 10.1002/14651858.CD003823.pub2]

\section{Heran 2008b}

Heran BS, Wong MMY, Heran IK, Wright JM. Blood pressure lowering efficacy of angiotensin receptor blockers for primary hypertension. Cochrane Database of Systematic Reviews 2008, Issue 4. Art. No: CD003822. [DOI: 10.1002/14651858.CD003822.pub2]

\section{Higgins 2019a}

Higgins JP, Thomas J, Chandler J, Cumpston M, Li T, Page MJ, et al, editor(s). Cochrane Handbook for Systematic Reviews of Interventions version 6.0 (updated July 2019). Cochrane, 2019. Available from www.training.cochrane.org/handbook.

\section{Higgins 2019b}

Higgins JP, Li T, Deeks JJ, editor(s). Chapter 6: Choosing effect measures and computing estimates of effect. In: Higgins JP, Thomas J, Chandler J, Cumpson M, Li T, Page MJ, et al, editor(s), Cochrane Handbook for Systematic Reviews of Interventions version 6.0 (updated October 2019). The Cochrane Collaboration, 2019. Available from www.training.cochrane.org/ handbook.

\section{Higgins 2019c}

Higgins JP, Savović J, Page MJ, Elbers RG, Sterne JA, editor(s). Chapter 8: Assessing risk of bias in a randomized trial. In: Higgins JP, Thomas J, Chandler J, Cumpston MS, Li T, Page MJ, et al, editor(s), Cochrane Handbook for Systematic Reviews of Interventions version 6.0 (updated October 2019). The Cochrane Collaboration, 2019. Available from www.training.cochrane.org/ handbook.

\section{Hunter 2019}

Hunter RF, de la Haye K, Murray JM, Badham J, Valente TW, Clarke $\mathrm{M}$, et al. Social network interventions for health behaviours and outcomes: a systematic review and meta-

Effectiveness of social network interventions to support cardiac rehabilitation and secondary prevention in the management of people 
analysis. PLoS Medicine 2019;16(9):e1002890. [DOI: 10.1371/ journal.pmed.1002890]

\section{Lefebvre 2019}

Lefebvre C, Glanville J, Briscoe S, Littlewood A, Marshall C, Metzendorf M-I, et al. Chapter 4: Searching for and selecting studies. In: Higgins JP, Thomas J, Chandler J, Cumpston M, Li T, Page MJ, Welch VA, editor(s). Cochrane Handbook for Systematic Reviews of Interventions Version 6.1 (updated September 2020). Cochrane, 2020. Available from www.cochrane-handbook.org.

\section{Lett 2005}

Lett HS, Blumenthal JA, Babyak MA, Strauman TJ, Robins C, Sherwood A. Social support and coronary heart disease: epidemiologic evidence and implications for treatment. Psychosomatic Medicine 2005;67(6):869-78.

\section{Liberati 2009}

Liberati A, Altman DG, Tetzlaff J, Mulrow C, Gøtzsche PC, loannidis JP, et al. The PRISMA statement for reporting systematic reviews and meta-analyses of studies that evaluate health care interventions: explanation and elaboration. PLOS Medicine 2009;6(7):e1000100.

\section{Michie 2011}

Michie S, van Stralen MM, West R. The behaviour change wheel: a new method for characterising and designing behaviour change interventions. Implementation Science 2011;6:42. [DOI: 10.1186/1748-5908-6-42]

\section{Mookadam 2004}

Mookadam F, Arthur HM. Social support and its relationship to morbidity and mortality after acute myocardial infarction: systematic overview. Archives of Internal Medicine 2004;164(14):1514-8.

\section{Piepoli 2016}

Piepoli MF, Hoes AW, Agewall S, Albus C, Brotons C, Catapano AL, et al. European Guidelines on cardiovascular disease prevention in clinical practice: the Sixth Joint Task Force of the European Society of Cardiology and Other Societies on Cardiovascular Disease Prevention in Clinical Practice (constituted by representatives of 10 societies and by invited experts). Developed with the special contribution of the European Association for Cardiovascular Prevention \& Rehabilitation (EACPR). European Heart Journal 2016;37(29):2315-81. [DOI: 10.1093/eurheartj/ehw106]

\section{Review Manager 2020 [Computer program]}

The Cochrane Collaboration Review Manager Web (RevMan Web). Version q.22.0. The Cochrane Collaboration, 2020. Available at revman.cochrane.org.

\section{Schünemann 2019}

Schünemann HJ, Higgins JP, Vist GE, Glasziou P, Akl EA, Skoetz N, et al. Chapter 14: Completing 'Summary of findings' tables and grading the certainty of the evidence. In: Higgins JP, Thomas J, Chandler J, Cumpston MS, Li T, Page MJ, et al, editor(s), Cochrane Handbook for Systematic Reviews of Interventions version 6.0 (updated October 2019). The Cochrane Collaboration, 2019. Available from www.training.cochrane.org/ handbook.

\section{SIGN 2017}

Scottish Intercollegiate Guidelines Network (SIGN). Cardiac rehabilitation: a national clinical guideline. www.sign.ac.uk/ media/1047/sign150.pdf (accessed prior to 16 October 2020).

\section{Simpson 2011}

Simpson SA, Shaw C, McNamara R. What is the most effective way to maintain weight loss in adults? BMJ 2011;343:d8042. [DOI: $10.1136 / \mathrm{bmj} . \mathrm{d} 8042]$

\section{Simpson 2015}

Simpson SA, McNamara R, Shaw C, Kelson M, Moriarty Y, Randell $E$, et al. A feasibility randomised controlled trial of a motivational interviewing-based intervention for weight loss maintenance in adults. Health Technology Assessment 2015;19:50. [DOI: 10.3310/hta19500]

\section{Simpson 2020}

Simpson SA, Matthews L, Pugmire J, McConnachie A, McIntosh E, Coulman E, et al. An app-, web- and social supportbased weight loss intervention for adults with obesity: the HelpMeDolt! feasibility RCT. Southampton (UK): NIHR Journals Library 2020 Mar. [DOI: 10.3310/phr08030]

\section{Stata [Computer program]}

Stata. Version 16. College Station, TX: StataCorp LLC, 2019. Available at www.stata.com.

\section{WHO 2017}

World Health Organization (WHO). Cardiovascular Diseases (CVDs). www.who.int/news-room/fact-sheets/detail/ cardiovascular-diseases-(cvds) 2017; (accessed prior to 05 November 2020).

\section{AP PE N DICES}

\section{Appendix 1. Preliminary MEDLINE Ovid search strategy}

1 exp Coronary Disease/ (218087)

2 ((coronary or heart) adj2 disease $\left.{ }^{\star}\right)$. tw. (259374)

3 CHD.tw. (25075)

4 CAD.tw. (39184)

5 exp Heart Failure/ (122114)

Effectiveness of social network interventions to support cardiac rehabilitation and secondary prevention in the management of people 
6 heart failure.tw. (166963)

7 HF.tw. (45717)

8 exp Myocardial Infarction/ (175710)

9 myocardial infarct ${ }^{\star}$. tw. (194875)

10 MI.tw. (47541)

11 heart attack*.tw. (5541)

12 Revasculari?ation*.tw. (57793)

13 exp Coronary Artery Bypass/ (52897)

14 coronary artery bypass.tw. (40608)

15 CABG.tw. (17997)

16 exp Percutaneous Coronary Intervention/ (54071)

17 percutaneous coronary intervention.tw. (30820)

18 PCl.tw. (25920)

19 exp Angioplasty/ (61553)

20 angioplast $^{\star}$. tw. (43465)

21 Angina, Stable/ (1351)

22 Stable angina.tw. (8058)

23 Atrial Fibrillation/ (55636)

24 atrial fibrillation*.tw. (70435)

25 AF.tw. (40412)

26 (valve adj2 (replace* or repair $\left.\left.{ }^{\star}\right)\right) . t w . ~(39487)$

27 or/1-26 (976828)

28 Social Networking/ (3529)

29 social network ${ }^{\star}$.tw. (17198)

30 exp social support/ (71403)

31 social support.tw. (38511)

32 Partner $^{\star}$.tw. (174598)

33 Caregivers/ (36925)

34 Caregiver $^{\star}$.tw. (64512)

35 Friends/ (5213)

36 Friend $^{*}$.tw. (88578)

37 Family/ (76201)

38 (family or families).tw. (948756)

39 Relatives.tw. (58102)

40 (spouse* or wife or wives or husband or husbands or partner ${ }^{\star}$ ).tw. (201765)

Effectiveness of social network interventions to support cardiac rehabilitation and secondary prevention in the management of people 
41 supporter ${ }^{\star} . t w .(3166)$

42 peer group/ (20653)

43 (peer or peers).tw. (92508)

44 Social Media/ (8137)

45 (social media or facebook or twitter).tw. (14503)

46 Mobile Applications/ (6149)

47 ((mobile or electronic) adj2 (app or apps or application*)).tw. (8155)

48 or/28-47 (1450097)

4927 and 48 (27663)

50 randomized controlled trial.pt. (512300)

51 controlled clinical trial.pt. (93824)

52 randomized.ab. (491073)

53 placebo.ab. (210651)

54 clinical trials as topic.sh. (192774)

55 randomly.ab. (340120)

56 trial.ti. $(224310)$

5750 or 51 or 52 or 53 or 54 or 55 or $56(1310665)$

58 exp animals/ not humans.sh. (4730611)

5957 not $58(1206602)$

6049 and $59(2383)$

\section{H I S T O RY}

Protocol first published: Issue 12, 2020

\section{CONTRIBUTIONSOF AUTHORS}

Carrie Purcell (CP): contributed to formulating the review question, led on drafting the protocol, approved and submitted final version of the protocol

Susie Smillie (SS): contributed to formulating the review question, contributed to drafting the protocol, approved final version for submission

Michele Hilton Boon (MHB): contributed to formulating the review question, reviewed and commented on the protocol, approved final version for submission

Sharon A Simpson (SAS): contributed to formulating the review question, reviewed and commented on the protocol, approved final version for submission

Rod S Taylor (RST): contributed to formulating the review question, reviewed and commented on the protocol, approved final version for submission

\section{DECLARATIONS OF INTEREST}

CP: none known

RT: none known

SS: none known

Effectiveness of social network interventions to support cardiac rehabilitation and secondary prevention in the management of people

Copyright (c) 2020 The Cochrane Collaboration. Published by John Wiley \& Sons, Ltd. 
SAS: none known

MHB: I am employed as a research associate by the MRC/CSO Social and Public Health Sciences Unit (SPHSU), University of Glasgow, to provide methodological support to the Cochrane Public Health and GRADE Public Health groups, and to conduct related methodological research. SPHSU is funded by unrestricted grants from the Medical Research Council and the Scottish Government Chief Scientist Office.

\section{SOURCES OF SUPPORT}

\section{Internal sources}

- $\mathrm{MRC} / \mathrm{CSO}$ Social and Public Health Sciences Unit, University of Glasgow, UK

Dr Purcell is currently funded by HRUK to undertake an implementation study of REACH-HF in Scotland. As this study is paused until Sept 2020 (due to COVID-19), it was agreed with HRUK that we would re-purpose part of her time to undertake this review. Ms Smillie and Dr Hilton Boon are supported by the Medical Research Council (grant numbers MC_UU_12017/14 and MC_UU_12017/15) and Chief Scientist Office of the Scottish Government Health and Social Care Directorates (grant numbers SPHSU14 and SPHSU15). Profs Taylor and Simpson are funded for their time as University of Glasgow employees.

\section{External sources}

- NIHR, UK

This project was supported by the National Institute for Health Research, via Cochrane Infrastructure funding to the Heart Group. The views and opinions expressed therein are those of the authors and do not necessarily reflect those of the Systematic Reviews Programme, NIHR, NHS or the Department of Health and Social Care. 